\title{
EVALUATION OF THE USE OF NON-SPECIFIC IMMUNOGLOBULINS IN BURNED PEDIATRIC PATIENTS
}

Betriu Sebastià $L^{1}$, Arévalo Bernabé $A G^{1}$, Lalueza Broto $\mathrm{P}^{1}$, Juárez-Giménez $\mathrm{JC}^{1}$, Cabañas Poy, $M \mathrm{JJ}^{1}$, Martínez de Compañón $Z^{2}$, Balcells Ramírez $\mathrm{J}^{2}$, Rossich Verdés $\mathrm{R}^{2}$ Servicio de Farmacia', Unidad de Cuidados Intensivos de Pediatría². Hospital Universitari Vall d'Hebron. Barcelona

\section{OBJETIVE}

- Serious burns produce plasma extravasation which develops loss of immunoglobulins (lg) and this could increase the risk of infections

- Evaluate the use and follow-up of the administration of non-specific Ig in burned pediatric patients

\section{METHODS}

- Population: Pediatric patients with $>10 \%$ burned surface area (BSA)

- Study period: Between August 2012 and March 2016.
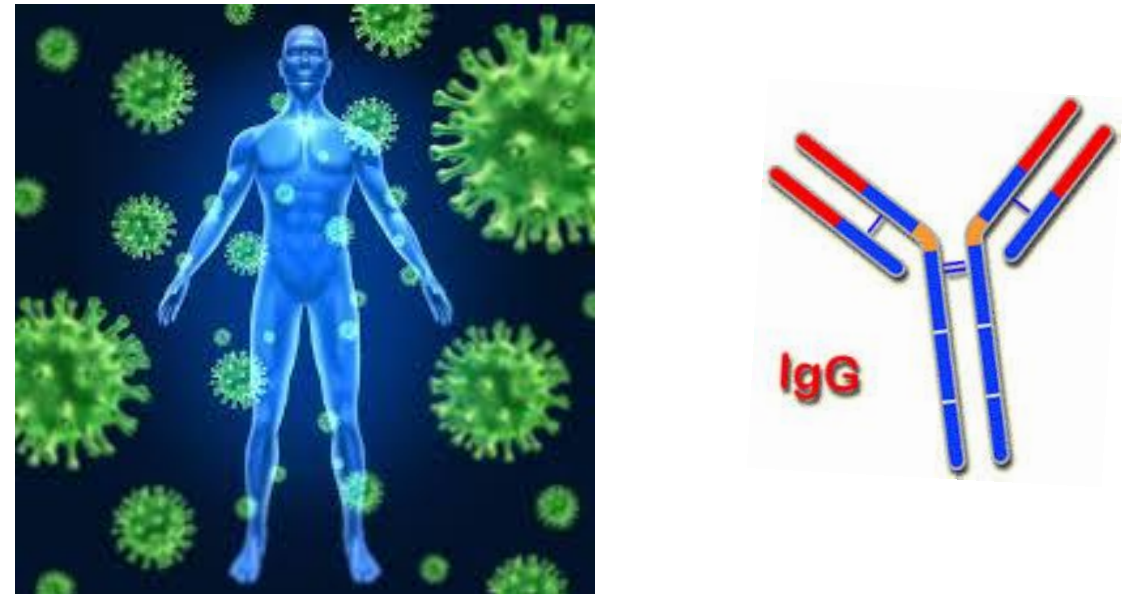

\section{RESULTS}

\begin{tabular}{|l|l|}
\hline \multicolumn{2}{|c|}{ BIODEMOGRAPHIC AND BURN DATA } \\
\hline Patients & $48(28$ ๙ $/ 20 \%)$ \\
\hline Age (years, median) & $2(8 \mathrm{~m}-15 \mathrm{y})$ \\
\hline Weight (Kg, median) & $12(7.5-67)$ \\
\hline BSA (\%, median) & $14.5(10-50 \%)$ \\
\hline \multirow{2}{*}{ Burn mechanism (patients, \%) } & Scald $\rightarrow 42(87.5)$ \\
\cline { 2 - 2 } & Fire $\rightarrow 5(10.4)$ \\
\cline { 2 - 2 } & Electricity $\rightarrow 1(2.1)$ \\
\hline
\end{tabular}

\begin{tabular}{|l|l|l|}
\hline \multicolumn{3}{|c|}{ Ig ADMINISTRATION DATA } \\
\hline $\begin{array}{l}\text { Determination of } \\
\text { IgG levels }\end{array}$ & $\begin{array}{l}37 \\
\text { PATIENTS } \\
(77 \%)\end{array}$ & $\begin{array}{l}\text { Below- normal levels: 25 } \\
\text { patients (67\%) with BSA 21\% } \\
(10-50 \%)\end{array}$ \\
\cline { 2 - 2 } & & $\begin{array}{l}\text { Normal levels: 12 patients } \\
(32 \%) \text { with BSA 12\% (10-15\%) }\end{array}$ \\
\hline $\begin{array}{l}\text { Number of } \\
\text { patients and dose } \\
\text { administered }\end{array}$ & $\begin{array}{l}25(52 \%) \text { and } 400 \mathrm{mg} / \mathbf{k g} \text { in all patients } \\
\text { enrolled }\end{array}$ \\
\hline $\begin{array}{l}\text { Moment of } \\
\text { administration }\end{array}$ & $\begin{array}{l}\text { - 48h post-burn : 12 patients (48\%) } \\
\text { - Subsequent days: 13 patients (52\%) }\end{array}$ \\
\hline
\end{tabular}

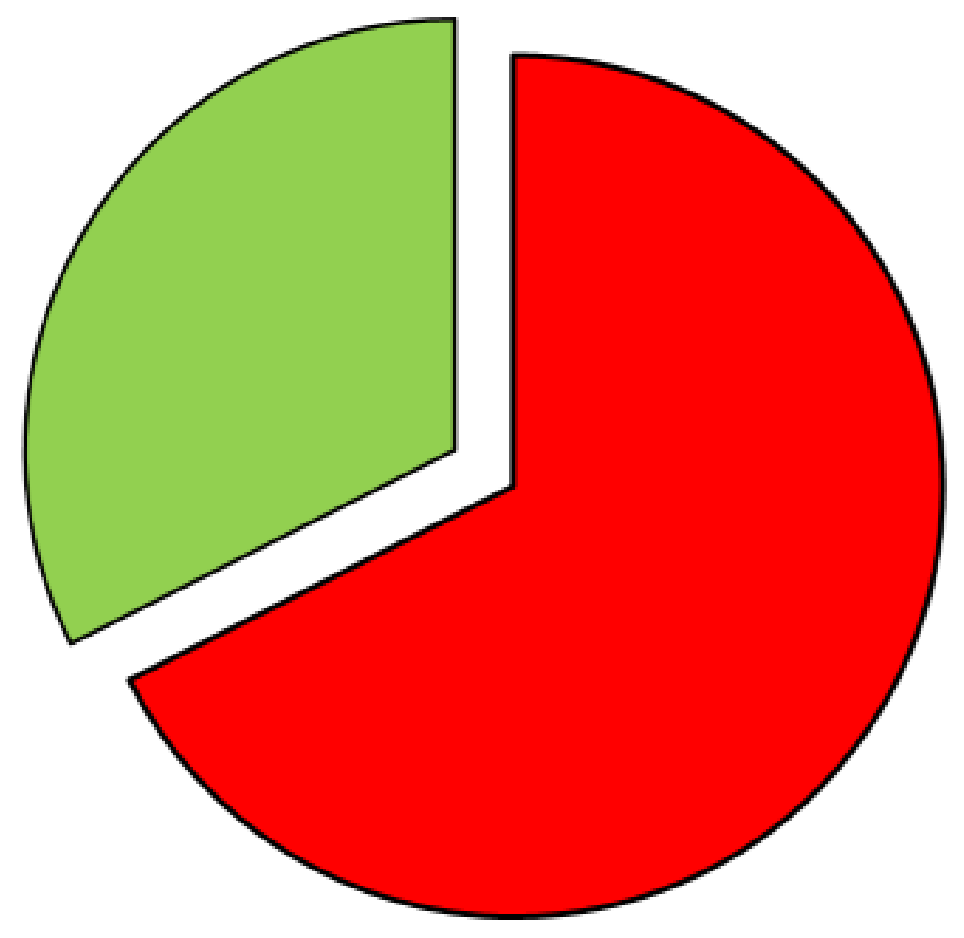

$\lg \mathrm{G}<400 \mathrm{mg} / \mathrm{dl} \quad$ BSA 21\%

$67 \%(25)$

\section{CONCLUSIONS}

$\checkmark$ There is a positive correlation between the BSA (20.5\%) and the depletion of immunoglobulin.

$\checkmark$ By analyzing the results, the authors recommend:

- The determination of IgG levels 72 hours post-burn, in children with BSA $>15 \%$.

- A new determination of $\lg G$ after a week, in patients with a $B S A>20 \%$ who have received an Ig infusion 